\title{
Study on Integrated Control of Active Anti-Roll Steel Bar and Electronic Stability Program Based on Particle Swarm Optimization
}

\author{
Chen Song ${ }^{1,2, ~ a, ~ L i ~ S h e n g y o n g, ~}{ }^{2, b}$, Sun $\mathrm{Xu}^{2, \mathrm{c}}$ \\ ${ }^{1}$ School of Automobile and Traffic Engineering, Jiangsu University, Zhenjiang 212013, China \\ ${ }^{2}$ Department of Transportation Engineering, Nantong Shipping College, Nantong 226010, China \\ achensong@ntsc.edu.cn, ${ }^{\mathrm{b}}$ Isy@ntsc.edu.cn, c'sunxuzh@ntsc.edu.cn
}

Keywords: Active anti-roll Steel bar, Rollover, Electronic stability program, Instability, Linear quadratic optimal, Particle swarm.

\begin{abstract}
Since a vehicle is prone to instability and rollover when taking a rapid turning, in this paper, an integrated control strategy of Electronic Stability Program (ESP for short) and active anti-roll steel bar (AARSB for short) is proposed. Then a three-degree-of-freedom vehicle dynamic model is established for rollover prevention and yaw stability control. In order to prevent the rollover and the instability of a vehicle, a linear quadratic (LQ for short) optimal controller is designed. The Particle Swarm Optimization (PSO for short) is adopted to optimize the weight coefficients of the LQ controller. Seven parameters are taken as the assessment criteria to carry out the simulation experiments on the vehicle with integrated control of AARSB and ESP in yaw stability and roll stability. These seven parameters are yaw rate, side-slip angle of mass center, roll angular velocity, roll angle, lateral load transfer ratio, additional yawing moment generated by ESP, and anti-roll moment generated by AARSB. To demonstrate the effects of the integration of AARSB and ESP on rollover prevention and instability control, two time typical simulations are conducted. The simulation results show that the vehicles with integrated control of AARSB and ESP can prevent the rollover and instability of a vehicle effectively. Therefore, with the integrated control, the driving stability of the vehicle is significantly improved.
\end{abstract}

\section{Introduction}

The driving safety of a vehicle greatly depends on the control over the yaw movement and the roll motion. The loss of yaw stability will lead to the loss of steering capability of a vehicle or will give rise to an intense turning of a vehicle. The instability and the rollover of a vehicle are dangerous traffic accidents happening frequently. As for the yaw stability control of a vehicle, the present major control methods proposed by the experts at home and abroad include the four-wheel steering control, the active suspension control, and the differential braking control, etc. As for the roll stability control of a vehicle, the current major control techniques are the active steering control, the active suspension control, the active anti-roll stabilizer bar control, and so on. However, the domestic and overseas study about the integrated control of the yaw stability and the roll stability is still in the initial exploration stage. Engineer MANDO proposed an integrated chassis control system composed of the semi-active suspension and the electronic stability program to control the rollover of a vehicle. The major strategy of the control system is, when there is a risk of a rollover monitored, the vehicle reduces the limiting lateral forces by putting a braking force on tires and by increasing the damping of the suspension shock absorbers to prevent the vehicle lurch [1]. Mohammad Kamal, from the Altair Engineering Inc, and Taehyun Shim, from the University of Michigan, using active suspension system, put forward a control strategy combined with the control over the instability and the control over the rollover of a vehicle [2]. They raised a concept of the Manipulative Model. That is, in the condition of large rollover index, the system will enter a pure rollover stability control mode; in the condition of small rollover index, the system will go into a pure yaw stability control mode. Besides, Germany BOSCH integrated the active front steering control system and the electronic stability control system to prevent a vehicle rollover [3]. Korean 
scholar Jangyeol Yoon et al., by tracking virtual test, designed a unified chassis control system combined with the electronic stability program and the active steering system [4]. K. JEON et al. utilized an electric control AARSB, established a single degree freedom dynamic model, and applied the method of sliding mode control to stop a vehicle rollover [5]. Bal'azs Varga et al. adopted LQ theory to calculate the required roll control torque of the hydraulically controlled AARSB for light commercial vehicles [6]. As the AARSB can change the roll angle stiffness of the suspension timely to prevent vehicles from rolling over according to the steering and driving conditions, this control technique, compared with several others, can be more direct and effective in rollover prevention. Additionally, the AARSB is low cost and easy to produce, so it has become a research hotspot. By adjusting the vertical load of the wheel, the AARSB can prevent the rollover of a vehicle, but simultaneously, the vehicle yaw stability will be influenced indirectly. And the ESP can generate additional yawing moment by putting different braking force to different wheels to control the yaw stability. In view of the case that a vehicle is prone to instability and rollover when taking a quick steering, this paper combines the AARSB and the ESP, and designs a linear quadratic (LQ) optimal controller to obtain the torque needed to keep the yaw stability and the roll stability of the vehicle. In that the LQ optimal controller has more weights, to apply the method of particle swarm optimization (PSO) can avoid subjectivity in designing.

\section{Vehicle Dynamic Model}
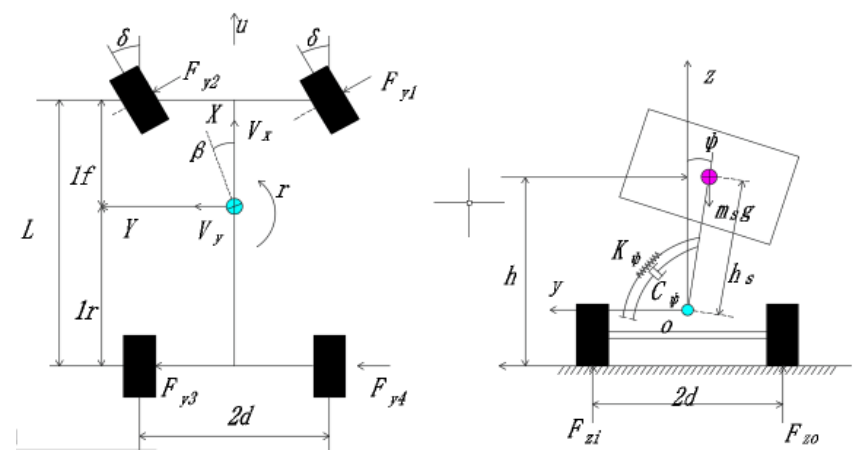

Fig. 1 DOF model for vertical and roll motions.

As shown in Fig. 1, a three-degree-of-freedom model is established for a vehicle. This model contains the yaw motion, the roll motion, and the lateral motion of the vehicle body. This is the three-degree-of-freedom model of the vehicle body.

The equation of lateral motion:

$$
m\left(\dot{v}_{y}+v_{x} \gamma\right)-\mathrm{m}_{\mathrm{s}} \mathrm{h} \ddot{\phi}=F_{f}+F_{r} .
$$

The equation of yaw motion:

$$
I_{Z} \dot{\gamma}+I_{X Z} \ddot{\phi}=l_{f} F_{f}-l_{r} F_{r}+M_{z} \text {. }
$$

The equation of roll motion:

$$
I_{x} \ddot{\phi}-m_{s} h_{s}\left(\dot{v}_{y}+v_{x} \gamma\right)+I_{x z s} \dot{\gamma}=m_{s} g h_{s} \phi-k_{\phi} \phi-c_{\phi} \dot{\phi}+U_{\text {anti-roll }} \text {. }
$$

In above equations,

$$
F_{f}=k_{f} \alpha_{f}, F_{r}=k_{r} \alpha_{r}, \alpha_{f}=-(\delta-\xi)=\beta+\frac{l_{f} \gamma}{v_{x}}-\delta, \alpha_{r}=\frac{v-l_{r} \gamma}{v_{x}}=\beta-\frac{l_{r} \gamma}{v_{x}}, \text { where } \quad \beta=\frac{v_{y}}{v_{x}}, \dot{\beta}=\frac{\dot{v}_{y}}{v_{x}} \text {. }
$$

The equations of motion are rewritten into the state equation as follow: 


$$
\left[\begin{array}{cccc}
m v_{x} & 0 & 0 & -m_{s} h \\
0 & I_{z} & 0 & I_{x z} \\
-m_{s} h v_{x} & I_{x z s} & 0 & I_{x s} \\
0 & 0 & 1 & 0
\end{array}\right]\left[\begin{array}{c}
\dot{\beta} \\
\dot{\gamma} \\
\dot{\phi} \\
\ddot{\phi}
\end{array}\right]+\left[\begin{array}{cccc}
-\left(k_{f}+k_{r}\right) & m v_{x}-\frac{k_{f} l_{f}-k_{r} l_{r}}{v_{x}} & 0 & 0 \\
-\left(k_{f} l_{r}-k_{r} l_{r}\right) & -\frac{k_{f} l_{f}{ }^{2}+k_{r} l_{r}^{2}}{v_{x}} & 0 & 0 \\
0 & -m_{s} h v_{x} & k_{\phi}-m_{s} g h & c_{\phi} \\
0 & 0 & 0 & -1
\end{array}\right]\left[\begin{array}{l}
\beta \\
\gamma \\
\phi \\
\dot{\phi}
\end{array}\right]=\left[\begin{array}{l}
-k_{f} \\
-k_{f} l_{r} \\
0 \\
0
\end{array}\right] \delta+\left[\begin{array}{ll}
0 & 0 \\
1 & 0 \\
0 & 1 \\
0 & 0
\end{array}\right]\left[\begin{array}{c}
M_{Z} \\
U_{\text {anti-roll }}
\end{array}\right]
$$

The equation can be further simplified as:

$$
\dot{x}=A x+B_{1} w+B_{2} u,
$$

where, $x=\left[\begin{array}{llll}\beta & \gamma & \phi & \dot{\phi}\end{array}\right]^{T} w=\delta, u=\left[\begin{array}{ll}M_{Z} & U_{\text {anti-roll }}\end{array}\right]^{T}$

$$
\begin{gathered}
A=-\left[\begin{array}{cccc}
m v_{x} & 0 & 0 & -m_{s} h \\
0 & I_{z} & 0 & I_{x z} \\
-m_{s} h v_{x} & I_{x z s} & 0 & I_{x s} \\
0 & 0 & 1 & 0
\end{array}\right]^{-1}\left[\begin{array}{cccc}
-\left(k_{f}+k_{r}\right) & m v_{x}-\frac{k_{f} l_{f}-k_{r} l_{r}}{v_{x}} & 0 & 0 \\
-\left(k_{f} l_{f}-k_{r} l_{r}\right) & -\frac{k_{f} l_{f}{ }^{2}+k_{r} l_{r}{ }^{2}}{v_{x}} & 0 & 0 \\
0 & -m_{s} h v_{x} & k_{\phi}-m_{s} g h & c_{\phi} \\
0 & 0 & 0 & -1
\end{array}\right] \\
B_{1}=\left[\begin{array}{cccc}
m v_{x} & 0 & 0 & -m_{s} h \\
0 & I_{z} & 0 & I_{x z} \\
-m_{s} h v_{x} & I_{x z s} & 0 & I_{x} \\
0 & 0 & 1 & 0
\end{array}\right]^{-1}\left[\begin{array}{l}
-k_{f} \\
-k_{f} l_{f} \\
0 \\
0
\end{array}\right], B_{2}=\left[\begin{array}{cccc}
m v_{x} & 0 & 0 & -m_{s} h \\
0 & I_{z} & 0 & I_{x z} \\
-m_{s} h v_{x} & I_{x z s} & 0 & I_{x} \\
0 & 0 & 1 & 0
\end{array}\right] \cdot\left[\begin{array}{cc}
0 & 0 \\
1 & 0 \\
0 & 1 \\
0 & 0
\end{array}\right] .
\end{gathered}
$$

In above expressions and Figures, $m$ is the mass of the vehicle body; $m_{s}$ is the sprung mass; $a_{y}$ is the lateral acceleration at the mass center; $\beta$ is the side slip angle; $h$ is the distance between the roll center and the mass center; $H$ is the height of center of gravity; $F_{f}$ and Fr are the lateral forces of the front wheels and the rear wheels respectively; $F_{L}$ and $F_{R}$ are the vertical roads on the left wheels and the right wheels; $k_{f}$ and $k_{r}$ are the front and the rear tire cornering stiffness; $\gamma$ is the yaw rate; $\varphi$ is the roll angle; $l_{f}$ and $l_{r}$ are the distance between the mass center and the front axle and the distance between the mass center and the rear axle respectively; $k_{\varphi}$ and $C_{\varphi}$ are the equivalence of the suspension roll stiffness and the equivalence of the damping coefficient; $v_{x}$ and $v_{y}$ are the longitudinal velocity and the lateral velocity; $T$ is the wheel tread; $I_{x}$ and $I_{z}$ are the moment of inertia around the $\mathrm{x}$-axis and the moment of inertia around the z-axis respectively; $I_{x z}$ is the product of inertia of the entire vehicle mass around the $\mathrm{x}$-axis and the $\mathrm{z}$-axis in the vehicle $\mathrm{z}$-axis coordinate system; $I_{x z s}$ is the product of inertia of the entire vehicle mass around the x-axis and the y-axis; $\alpha_{f}$ and $\alpha_{r}$ are the side slip angle of the front wheel and that of the rear wheel; $\delta$ is the steering angel of the front wheels; $M_{z}$ is the additional yaw moment; $U_{\text {anti-roll }}$ is the anti-roll moment generated by the AARSB.

\section{Controller Design with LQ PSO}

LQ Optimal Controller. When taking a sharp turning, the vehicle is prone to rollover and instability. In order to reduce the rollover of the vehicle, it is needed to control the roll angular velocity, the roll angle and the lateral load transfer ratio output from the vehicle. And in order to reduce the instability of the vehicle, it is required to control the vehicle's yaw rate and side-slip angle of mass center. And also, it is requisite to simultaneously consider the electrical energy consumption from the AARSB by providing an anti-roll moment and an additional yaw moment as small as possible. In view of the above, the performance index is:

$$
J=\int_{0}^{\infty}\left\{\rho_{1}\left(\beta-\beta_{d}\right)^{2}+\rho_{2}\left(\gamma-\gamma_{d}\right)^{2}+\rho_{3} \phi^{2}+\rho_{4} \dot{\phi}^{2}+\rho_{5} I^{2}{ }_{\text {LTR }}+\rho_{6} M_{z}^{2}+\rho_{7} U_{\text {anti-roll }}{ }^{2}\right\} d t .
$$

The value range of $I_{L T R}$ is [-1 1 ]. If $I_{L T R}=0$, the vertical load on the left wheel is equal to that on the right; if $I_{L T R}= \pm 1$, it means that the vertical load on one side is zero, and that the vehicle rolls over. $\gamma_{d}$ and $\beta_{d}$ are respectively the nominal yaw rate and the nominal side-slip angle of mass center 
under an ideal two-degree freedom model. The control of the vehicle instability is by letting the yaw rate and side-slip angle of mass center of the vehicle with AARSB track the nominal yaw rate and the nominal side-slip angle under the ideal two-degree freedom model. When there are deviations between the actual value and the nominal value, according to the deviation, the ESP ensures the vehicle yaw stability by applying the method of differential braking on the left and the right wheels to obtain the additional yawing moment needed. The control objective of the ideal model is a zero side slip angle, that is, $\beta_{d}=0$. At that time, the nominal yaw rate under the ideal two-degree freedom model is as follow:

$$
\gamma_{d}=\frac{k_{f} k_{r}(a+b) v_{x}}{k_{f} k_{r}(a+b)^{2}+m v_{x}^{2}\left(\mathrm{~b} k_{r}-a k_{f}\right)} \delta \text {. }
$$

Then the output variable can get from the expression:

$$
y=C x+D_{1} w+D_{2} u
$$

In the expression (8), $\mathbf{y}=\left[\left(\beta-\beta_{d}\right),\left(\gamma-\gamma_{d}\right), \phi, \dot{\phi}, I_{L T R}, M_{z}, U_{\text {anti-roll }}\right]^{T}$

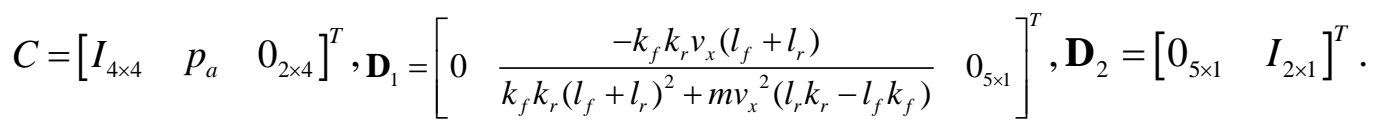

In these expressions:

$$
p_{a}=\left[\begin{array}{llll}
\frac{2 H I_{x}\left(k_{f}+k_{r}\right)}{T g\left(m I_{x}-m_{s}{ }^{2} h^{2}\right)} & \frac{2 H I_{x}\left(k_{f} l_{f}-k_{r} l_{r}\right)}{T g v_{x}\left(m I_{x}-m_{s}{ }^{2} h^{2}\right)} & \frac{2 H\left(m_{s}{ }^{2} h^{2} g-m_{s} h k_{\phi}\right)}{T g\left(m I_{x}-m_{s}{ }^{2} h^{2}\right)}+\frac{2 h}{T} & -\frac{2 H m_{s} h c_{\phi}}{T g\left(m I_{x}-m_{s}{ }^{2} h^{2}\right)}
\end{array}\right] .
$$

Put the Eq. (8) into the expression (6), the performance index turns into as follows:

$$
J=\frac{1}{2} \int_{0}^{\infty}\left[X^{T} Q X+2 X^{T} N U+U^{T} R U\right] d t,
$$

where, $Q=C^{T} q C$. $Q=C^{T} q C$ is the weight matrix of state variable. $R=D_{1}^{T} q D_{1}+r$ is the weight matrix of controlled inputs. $R=D_{1}{ }^{T} q D_{1}+r$ and $N=C^{T} q D_{1}$ are the weight matrixes of cross-term. $q=$ $\operatorname{diag}\left(\boldsymbol{q}_{1} \boldsymbol{q}_{2} \boldsymbol{q}_{3} \boldsymbol{q}_{4} \boldsymbol{q}_{5}\right), q_{1}=\boldsymbol{\rho}_{1}, q_{2}=\boldsymbol{\rho}_{2}, q_{3}=\boldsymbol{\rho}_{3}, q_{4}=\boldsymbol{\rho}_{4}, q_{5}=\boldsymbol{\rho}_{5}, r=\operatorname{diag}\left(\boldsymbol{r}_{1} \boldsymbol{r}_{2}\right), \boldsymbol{r}_{1}=\boldsymbol{\rho}_{6}, \boldsymbol{r}_{2}=\boldsymbol{\rho}_{7}$. And the optimal control law is:

$$
\boldsymbol{U}=-\boldsymbol{K} \boldsymbol{X} .
$$

In this paper, the particle swarm optimization (PSO) is used to find the optional value of $K$.

Particle Swarm Optimization (PSO) of Control Parameters. The selection of weighting matrix $Q$ and $R$ is the key work of designing an LQ optimal controller [7]. If the LQ optimal controller has more weights, to apply the method of PSO can save time and avoid subjectivity in designing.

PSO is a biological evolutionary algorithm. Supposing the position and the velocity of the $i$-th particle are $x i=(x i, 1 \quad x i, 2 \cdots x i, d)$ and $v i=(v i, 1 \quad v i, 2 \cdots v i, d)$ respectively, where the letter $d$ stands for the number of the target optimization variables. In the process of each iteration, particles update themselves by tracking two optimal solutions. One optimal solution, $p i=(p i, 1 p i, 2 \cdots p i, d)$, is from the iteration of the particle itself, whose fitness value is recorded as pbest; the other optimal solution, $p g=(p g, 1 p g, 2 \cdots p g, d)$, is found currently from the iteration of all the particles, whose fitness value is recorded as gbest. Then, the PSO with the optimization objects and the constraints of the controller weights are written in the Matlab program. The operating parameters of particle swarm optimization can be listed as follows: particle population size is set to 32; particle size is set to 10; assign 100 to the maximum of iterations; make the learning factor $c_{1}=c_{2}=2$, the minimum inertia weight factor $w_{\min }$ and the maximum inertia weight factor $w_{\max }$ are respectively set 0.35 and 0.85; and make the maximum particle searching velocity be $20 \%$ of the global particle range. Fig. 2 is the adaptive value iteration curve of PSO. The values of $\rho_{1}, \rho_{2}, \rho_{3}, \rho_{4}, \rho_{5}, \rho_{6}$, and $\rho_{7}$ are obtained as $80.6020,40.1034,1.6536 \mathrm{e}+04,2.2135 \mathrm{e}+05,30,1.0000 \mathrm{e}-06$, and $1.0000 \mathrm{e}-06$ respectively. 


\section{Simulations and Results Analysis}

Fish Hook Simulation. In order to verify the anti-roll effect of the integrated control of AARSB and ESP optimized by PSO, the simulation with fish hook input is employed. The velocity is $80 \mathrm{~km} / \mathrm{h}$, and the attachment coefficient of the road surface is 0.8 . For the sake of comparing, a passive system without any control (be shown as Open Loop in the figure below), an active system only controlled with AARSB (be shown as AARSB in the figure below), a system only controlled with ESP (be shown as ESP in the figure below), and an integrated control system of AARSB and ESP have been carried out respectively in simulation. Fig. 2 and Fig. 3 show that in rollover prevention, the vehicle, controlled with integrated system or only controlled with AARSB, can effectively reduce the roll angle and the roll angular velocity. From Fig. 4, it is illustrated that the vehicle with passive anti-roll bar has a risk of rollover because the lateral load transfer ratio is close to 1 , while the vehicle with AARSB, whose maximum of the lateral load transfer ratio is less than 0.6 , demonstrates a better capacity of rollover prevention. Because the control of yaw stability with ESP is carried out by the method of differential braking, and because the method of differential braking shows certain preventative effects in rollover control, to adopt the integrated control of ESP and AARSB manifests a better effects than the effect of only to adopt AARSB to prevent a vehicle rollover, which can be demonstrated in Fig. 2, Fig. 3 and Fig. 4.

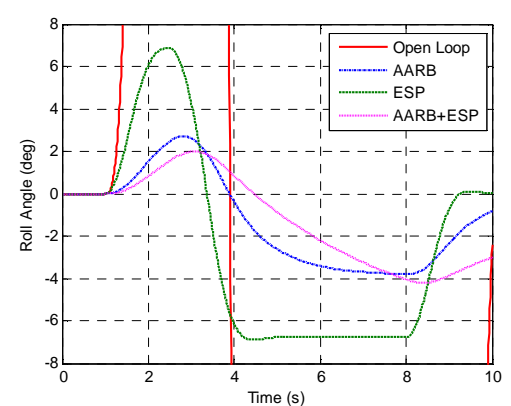

Fig. 2 Roll angle.

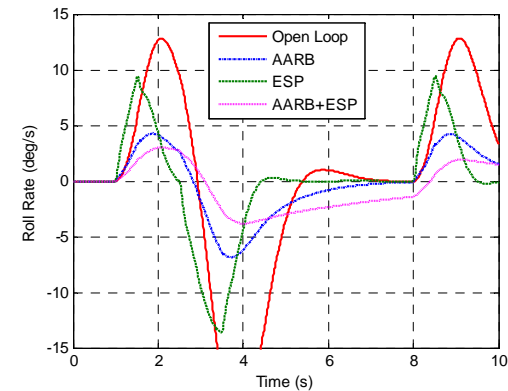

Fig. 3 Roll rate.

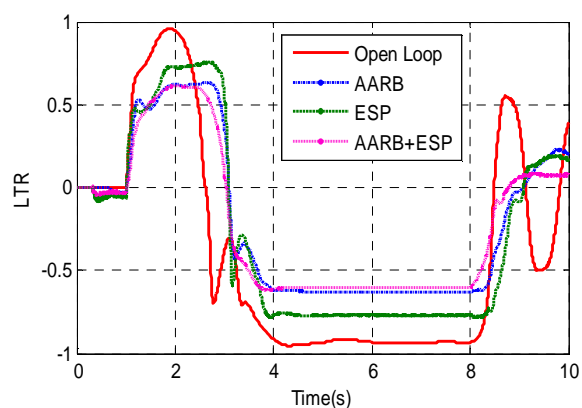

Fig. 4 Lateral load transfer ratio.

Emergency Double Lane Change Simulation. Among the vehicle steering stability experiments under extreme working conditions, the emergency double lane change simulation is frequently conducted by scholars and research institutions at home and abroad. In this paper, the emergency double lane change simulation is also employed to test the vehicle steering stability based on the integrated control of AARSB and ESP. The velocity is $55 \mathrm{~km} / \mathrm{h}$, and the simulation is conducted on the icy road, the attachment coefficient of whose surface is 0.15. Fig. 5 and Fig. 6 show that in yaw stability control, the vehicle, controlled with AARSB or with passive system, can not reduce the yaw rate and side-slip angle of mass centre effectively, and even relatively high amplitude fluctuations may take place. So the vehicle deviates from the ideal traveling track of the drive, and instability occurs, which can be displayed in Fig. 7. And due to the increased lateral load transfer of the left and the right wheels, which causes the change of the lateral force of the inner and the outer wheels when the AARSB controls the rollover of a vehicle, the vehicle controlled with AARSB even has a poor effect in yaw stability. However, the vehicle employed the integrated control system of AARSB and ESP or that employed ESP can well control the yaw rate and side-slip angle of mass centre, and can successfully track the ideal travel trace of the driver. So the two systems have a sound effect in vehicle stability control. Because of the indirect effect on the yaw stability of the integrated control of AARSB and ESP during the vehicle rollover control by AARSB, the control result of employing ESP only is slightly superior to the result of the integrated control in yaw stability, which can be illustrated in Fig. 5, Fig. 6 and Fig. 7. 


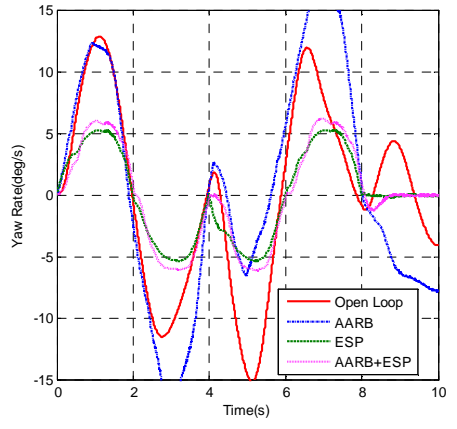

Fig. 5 Yaw rate.

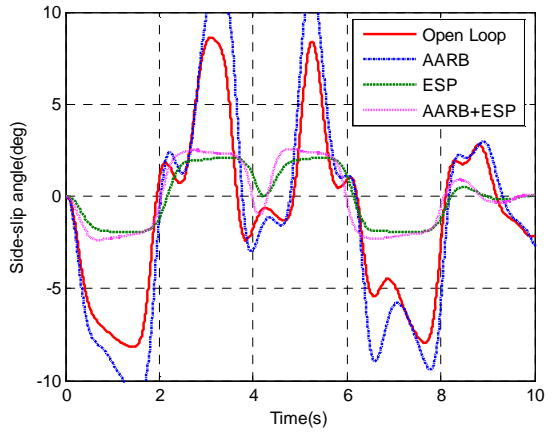

Fig. 6 Side-slip angle of mass center.

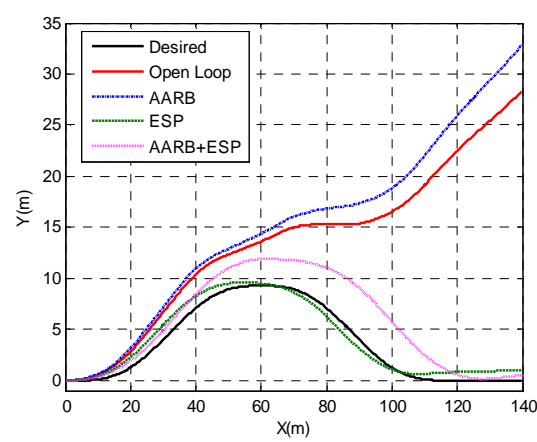

Fig. 7 Travel track.

To analyze the simulation results in fish hook and in double lane change synthetically, compared to other control systems, the integrated system of AARSB and ESP takes obvious advantages in rollover prevention and steering stability. Thus, the integrated control system can ensure the vehicle driving safety effectively.

\section{Conclusion}

(1) Since a vehicle is prone to instability and rollover when taking a rapid turning, an integrated control strategy of AARSB and ESP is proposed in this paper.

(2) The simulation results demonstrate that the vehicles with integrated control of AARSB and ESP can prevent the rollover and instability of a vehicle effectively. Therefore, with the integrated control system, the driving safety of the vehicle is significantly improved.

\section{References}

[1] J. Y. Yoon, W. K. Cho, B. Y. Koo, K. S. Yi, Unified Chassis Control for Rollover Prevention and Lateral Stability, Vehicular Technology, IEEE Transactions, 2009.

[2] K. Mohammad, T. Y. Shim, Development of Active Suspension Control for Combined Handling and Rollover Propensity Enhancement, SAE Paper, 2007-01-0826, 2007.

[3] K. Willy, K. Martin, S. Wolfgang, Integrated Safety Concept and Design of a Vehicle Dynamics Management System, In: SAE Technical Paper, Detroit, 2007-01-0842, 2007.

[4] J. Y. Yoon, W. K. Cho, J. Y. Kang, et al. Design and evaluation of a unified chassis control system for rollover prevention and vehicle stability improvement on a virtual test track, Contr. Eng. Pract. 18(6) (2010) 585-597.

[5] K. Jeon, H. Hwang, S. Choi, etc. Development of an electric active rollcontrol (arc) algorithm for a suv. Int. J. Autom. Tech. 13(2) (2012) 247-253.

[6] B. Varga, B. N’emeth, P. G'asp'ar. Control Design of Anti-Roll Bar Actuator Based on Constrained LQ Method. CINTI 2013. 14th IEEE International Symposium on Computational Intelligence and Informatics. 19-21 November, 2013. Budapest, Hungary 31-36.

[7] Y. Q. Guo, Z. D. Xu, S. M. Fei, et al. PSO control used in magnetorheological intelligent structures, J. Vib. Shock 30(9) (2011) 59-63. 